\title{
Design of Robust Current Tracking Control for Active Power Filters
}

\author{
Z. Y. Wu, N. Schofield, C. M. Bingham, D.Howe and D. A. Stone \\ Department of Electronic \& Electrical Engineering \\ University of Sheffield, Mappin Street, Sheffield S1 3JD, UK \\ E-mail: s.j.gawthorpe@shef.ac.uk
}

\begin{abstract}
The paper describes a design methodology for robust current-tracking control of active power filters using quantitative feedback theory (QFT). The design aim is to address system issues of power quality and power factor correction in a double-sided converter (rectifier/inverter combination) subject to parametric uncertainty, non-linear dynamic behavior and exogenous disturbances. The paper includes simulation results to demonstrate the dynamic performance attributes afforded to the resulting closed-loop control system, and to verify the design procedure.
\end{abstract}

Keywords: Active power filter, two-degree-of-freedom, QFT, DSP.

\section{INTRODUCTION}

The increasing demand for high-power electronic converters to be connected directly to finite-impedance AC utility supplies (power utility or stand-alone generator) and increasingly stringent regulations regarding harmonic distortion is focusing attention on cost effective methods for mitigating problems associated with harmonic. In addition to distortion of the voltage waveform, harmonic currents cause additional heating, and can result in over-voltages due to resonances, metering errors, malfunctions of protection relays and interference with communication and control signals, etc. Furthermore, phase-controlled converters are known to create 'notches' in the utility voltage waveform and draw power at a relatively poor displacement (power) factor.

Traditionally, economic and technological constraints have meant that equipment manufacturers have addressed these problems by incorporating a passive RLC filter at the input to each converter. However, with recent reductions in the cost of switching devices and the availability of inexpensive, digital processing hardware, embedded approaches to actively reduce the generation of high current harmonics onto the supply, are attracting increasing attention.

A typical topology for an active power filter is the so-called doubled-sided converter, shown in Figure 1 . The line input is generally from the power utility or a stand-alone generator. Due to its symmetrical structure, the line-side converter can act as either a rectifier or an inverter, thereby allowing bidirectional power flow by using appropriate pulse-width modulation (PWM) techniques.

The current control system is the primary component of all active power filtering systems; the basic objectives being,

(i) To reduce amplitude and phase errors (i.e. ideal tracking) between the reference and output currents over the specified output frequency range;

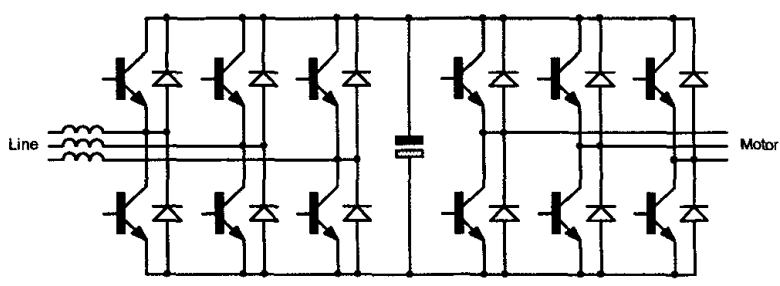

Figure 1. Double-sided converter, combining active power filter and voltage source converter.

(ii) High bandwidth, in order to provide a good dynamic system performance;

(iii) Limited variation of the switching frequency, so as to ensure safe operation of the power switching devices; and

(iv) Good utilization of the $\mathrm{dc}$ link voltage, to accommodate a wide speed range of the generator and/or load machine.

The simplest current control scheme is hysteresis control. However, the switching frequency then depends largely on the load parameters and hysteresis band, and may vary widely. Thus, currently, for most commercial applications, fixed frequency PWM current controllers, based on linear PIregulator control constructs, are employed. However, whilst these are particularly suitable for tracking steady-state quantities, when applied to tracking sinusoidal inputs, they naturally impart phase errors (as a classical frequency response characteristic will show), a feature that is particularly detrimental to the requirement for unity power factor operation. Appropriately designed tracking compensators are, therefore, necessary. Many authors have recently proposed controller design methodologies for active filters, one of the more promising being that proposed in [1], which employs internal model control, which is shown to provide a degree of robustness to parametric uncertainties. However, the controller was not designed specifically for current tracking, and important issues such as the rejection of system input and output disturbances, and non-linearities associated with the converter and its modulation control, were not directly addressed.

In this paper, quantitative feedback theory (QFT) is employed to design the current tracking controllers. Design difficulties stemming from system uncertainties, such as line filter parameter variations, non-linearities due to overmodulation of the voltage source inverter and switching of the power components, system output disturbances due to current harmonics, and system input disturbances from d.c. link 
voltage fluctuations etc., are either explicitly or implicitly considered in the design of the controller.

A schematic of the proposed two-degree-of-freedom control system is shown in Figure 2.

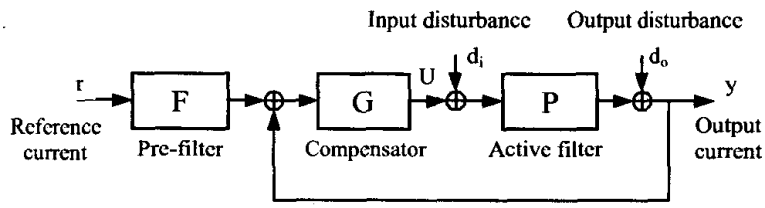

Figure 2. Two-degree-of-freedom current control system.

\section{QUANTITATIVE FeEDBaCK THEORY (QFT)}

As an extension to the pioneering work of Bode, Nyquist, Nichols, and others, Horowitz [2] introduced a frequency domain-design methodology which was refined in the 1970's to its present form, commonly referred to as Quantitative Feedback Theory (QFT), [3]. The underlying principles of two-degree-of-freedom QFT problem is to 'tune' the amount of inner-loop gain to accommodate specific amounts of plant and disturbance uncertainty, whilst the pre-filter is used to provide the desired 'shape' of the closed-loop dynamics. Although other two-degree-of-freedom design methodologies have attracted substantial attention over the past decade (e.g. $\mathrm{H}_{\infty}$ ), QFT has the advantage of leaving the designer to make the quantitative trade-off between performance and controller complexity. Based on the properties of the Nichols chart, the underlying principle of the technique consists of obtaining a set of frequency-domain boundaries about a nominal loop transfer function. These bounds are then used as a guide for shaping the loop transfer function, and, in so doing, to also accommodate any uncertainty.

To date, a major impediment to the adoption of QFT for control system design has been the graphical manipulation required during the design process, and the significant experience required of the designer. However, the emergence of a PC-based graphical QFT toolbox [4] has been instrumental in attracting a wider audience to the merits of QFT.

The design methodology generally involves four basic steps, viz.: (1) definition of problem data and generation of plant templates, (2) computation of QFT bounds, (3) design of controller and pre-filter (for tracking controllers) using loop-shaping techniques, and (4) analysis validation of the resulting design.

\section{Modeling of Active PoWer Filter}

Each input line of the active power filter can be modeled as a resistance connected in series with an inductance. If it is assumed that the power drawn from the supply has unity power factor, and that the phase voltage is controlled by the modulation depth $m$ in the PWM modulator, the open-loop transfer function which relates the phase current to the modulation depth reduces to,

$$
P(s)=\frac{m(s)}{i(s)}=\frac{K}{R+L s}
$$

where $R$ and $L$ are, respectively, the resistance and inductance of the line boost inductor, and $K$ is the voltage factor relating the d.c. link voltage to the PWM modulation depth.

Practically, $L$ and $R$ are known only to a limited accuracy, an error within $\pm 10 \%$ being very common. They are also dependent on frequency and operating condition, and it is estimated that in the frequency range of interest, viz. up to $1 \mathrm{kHz}$, the inductance could decrease by around $7 \%$, whilst the resistance could increase by a factor of 10 . Table 1 gives typical values of these two parameters in the application under consideration.

Table 1. Typical parameter uncertainties in the active power filter.

\begin{tabular}{|c|c|c|}
\hline $\begin{array}{c}\text { Inductance } L \\
(\mu H)\end{array}$ & $\begin{array}{c}\text { Resistance } R \\
(m \Omega)\end{array}$ & $\begin{array}{c}\text { Voltage factor } \\
K\end{array}$ \\
\hline $180 \sim 220$ & $7.2 \sim 72.0$ & $180 \sim 240$ \\
\hline
\end{tabular}

In double-sided converters, from the a.c. sides both converters appear as buck converters. Thus, the d.c. link voltage has to be higher than the peak values of both the line and load voltages. In electrical drive applications, the dc-link voltage is allowed to vary within a bounded range during motoring and regenerative braking of the machine, whilst in others, such as those incorporating super-capacitor energy storage, a tunable d.c. link voltage is beneficial to overall system energy efficiency. Such a fluctuating d.c. link voltage may be regarded as an input disturbance to the current control system. However, it can be more conveniently considered as a parameter uncertainty in the QFT design. A typical variation of the voltage factor in an active power filter is shown in Table 1, assuming that the modulation remains in the linear region.

In addition to the parametric uncertainties in the system, non-linearities stemming from the switching characteristic of the power switching devices, and saturation non-linearity due to over-modulation, are also present. However, whilst the former may be generally regarded as parameter uncertainty in the voltage factor, the latter has to be dealt with carefully at the control design stage, as will be discussed later.

Other disturbances include imperfections in the modulation algorithm and the resultant current harmonics in the output.

\section{Robust CURRENT TraCKING CONTROL DESIGN}

The control objective is to synthesize the pre-filter, $F(s)$, and the inner-loop compensator, $G(s)$, in the two-degree-offreedom feedback configuration such that, with minimal control effort, the resulting system provides the desired current tracking capability, and good disturbance attenuation performance. 


\section{A. Current Control Performance Specification}

1) Robust Stability: The current control system must maintain its stability properties over the full range of parametric uncertainty. To accommodate this, a constraint on the peak magnitude of the complementary sensitivity function for the range of potential open-loop transfer functions (described by templates), can be employed. For example, the requirement

$$
\left|\frac{P(j \omega) G(j \omega)}{1+P(j \omega) G(j \omega)}\right|<1.2, \quad \text { for } \omega>0, \forall P \in \text { template }
$$

implies that at least a $50^{\circ}$ phase margin and at least 1.8 gain margin is desired.

2) Current Tracking: Tracking performance can be satisfied by setting upper and lower bounds on the closedloop time-domain transient characteristic. Normally, an under-damped time response is specified for the upper bounds, e.g. with an overshoot of $20 \%$, and a rise time of $0.5 \mathrm{~ms}$ corresponding to the required $1 \mathrm{kHz}$ current tracking. An over-damped time response would be suitable for the lower bound, and again, the rise time is set at $0.5 \mathrm{~ms}$. Secondorder transfer functions which describe the upper and lower bounds in the frequency-domain are:

$$
\begin{aligned}
& T_{R_{U}}=\frac{1.04 \times 10^{4}(s+2000)}{s^{2}+9000 s+2.08 \times 10^{7}} \\
& T_{R_{L}}=\frac{2.56 \times 10^{8}}{s^{2}+32000 s+2.56 \times 10^{8}}
\end{aligned}
$$

\{Note: In order to extend the spread between the upper and lower bounds in the high-frequency range, a zero is introduced in $T_{R_{U}}$ without unduly affecting the desired timeresponse characteristics.\}

Since the plant is minimum phase, a tolerance on the magnitude is sufficient for design:

$$
\begin{aligned}
\left|T_{R_{L}}(j \omega)\right| \leq\left|\frac{F(j \omega) G(j \omega) P(j \omega)}{1+G(j \omega) P(j \omega)}\right| \leq\left|T_{R_{U}}(j \omega)\right|, & \text { for } \omega \in[0,6283]
\end{aligned}
$$

3) Control Effort Constraint: PWM control of a voltage source converter in an active power filter has a limited range of operation. For example, if space vector PWM is employed, the linear control range can be up to 1.15 . However, for generality this paper considers a simple PWM algorithm with a linear control range from 0 to 1 . Any value beyond this will push the PWM into over-modulation, i.e. the PWM control will introduce a hard saturation non-linearity which may degrade the system tracking performance, and should, therefore, be avoided in the design over the frequency range of interest. The worst case scenario is when the converter supplies its maximum rated current, i.e. $90 \mathrm{Amps}$, and the
PWM control reaches its saturation level of 1 . Thus, the following constraint is imposed:

$$
\left|\frac{G(j \omega)}{1+G(j \omega) P(j \omega)}\right|<0.011, \text { for } \omega<6283 \mathrm{rad} / \mathrm{s}
$$

4) Output Disturbance Attenuation: Due to the limited switching frequency of the converter power components, and imperfections in the PWM, there will inevitably be some current harmonics at the output that will constitute an output disturbance to the control system. Consequently, a constraint on the magnitude of the sensitivity function is included in the design,

$$
\left|\frac{1}{1+G(j \omega) P(j \omega)}\right|<\left|1.6 \times 10^{-4} \omega\right|, \quad \text { for } \omega<6283 \mathrm{rad} / \mathrm{s}
$$

It should be noted that this specification requires the system to have infinite attenuation at low frequencies, and thereby necessitates the inclusion of integral action.

\section{B. Robust Current Control Design}

A QFT-based controller design will now be expounded to satisfy the previously highlighted performance requirements. The performance specifications are consequently translated onto magnitude vs. phase plots (i.e. Nichols charts), and subsequently combined into a single set of "worst case" performance boundaries, Figure 3 (using facilities available in the QFT-toolbox). The objective of the design is to loopshape $G(j \omega) P(j \omega)$, by appropriate choice of the compensator $G(j \omega)$ and pre-filter $F(j \omega)$, such that at each frequency the magnitude and phase-shift of the nominal loop transfer function lies 'above' or 'outside' the respective performance boundary.

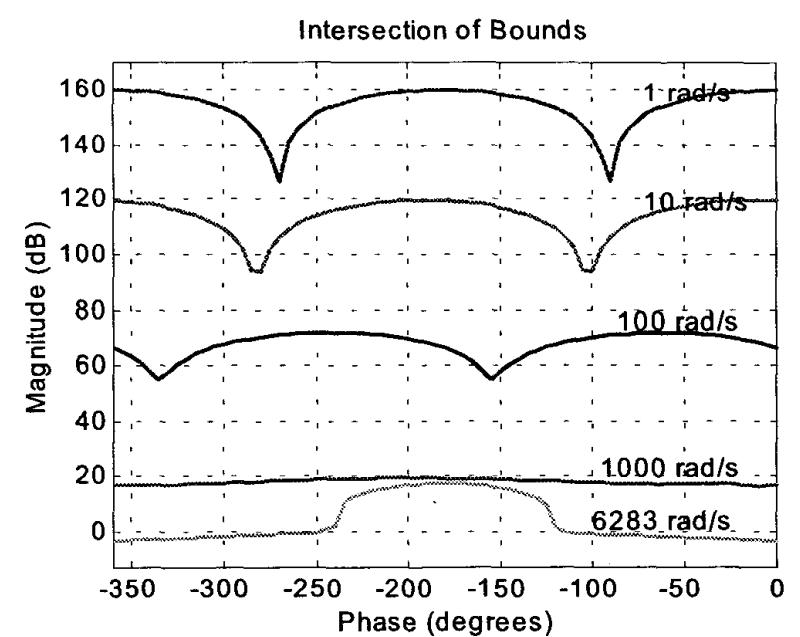

Figure 3. Combined performance bounds of robust control system. 
1) Compensator Design: The initial step of the design is to close the loop by designing the compensator $G(s)$ (assuming $F(s)=1$ ). Loop-shaping is employed to design an appropriate compensator to satisfy the combined boundary constraints shown in Figure 3. The result of an iterative loopshaping procedure is shown in Figure 4, which indicates that the system is stable and all the desired performance attributes are satisfied. The resulting compensator is:

$$
G(s)=\frac{(s+390)(s+1125)}{90 s(s+18)}
$$

2) Pre-Filter Design: Following the design of $G(s)$, the design of the pre-filter $F(s)$ to meet the dynamic tracking performance specification is addressed, resulting in the closed-loop frequency response shown in Figure 5. The prefilter is given by:

$$
F(s)=\frac{2.651 \times 10^{8}}{(s+6400)\left(s+4.143 \times 10^{4}\right)}
$$

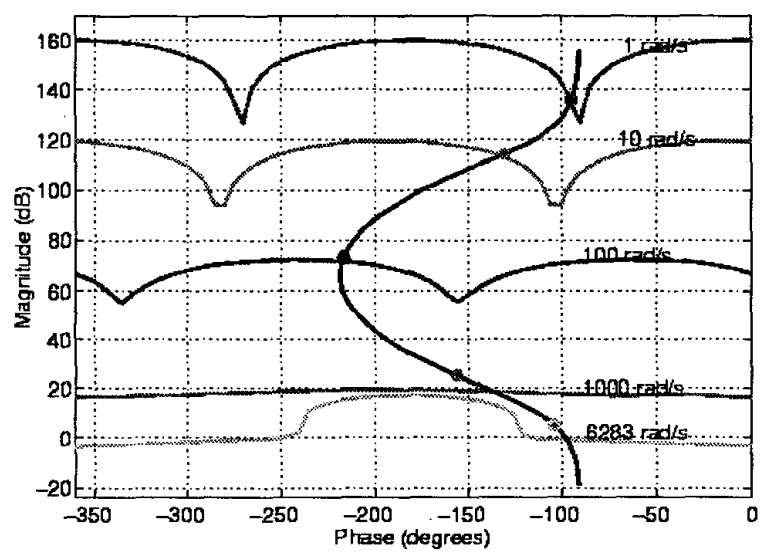

Figure 4. QFT loop-shaping design to satisfy robust performance bounds.

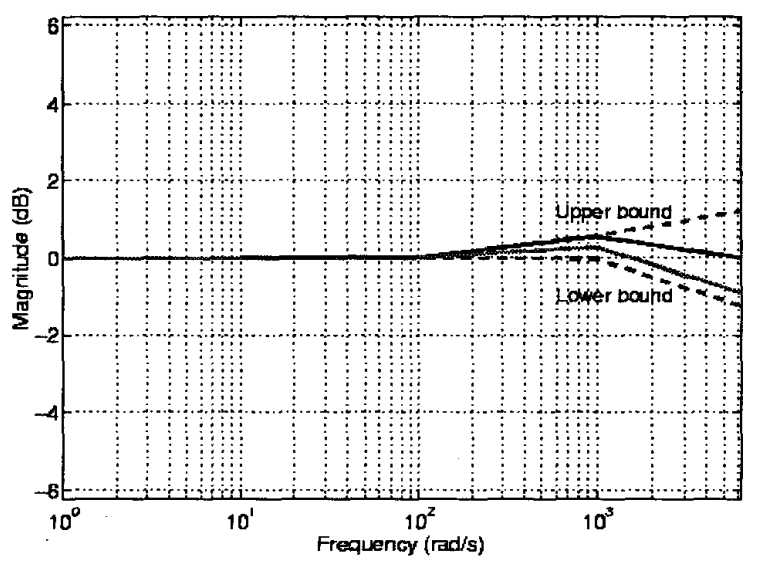

Figure 5. QFT design of pre-filter to satisfy tracking performance.

\section{COMPUTER SIMULATIONS}

The resulting control system design and its specification have been verified by computer simulations. In addition, the performance of the controller has been compared with that of a 'conventionally-designed' PI compensator.

\section{A. Computer Simulations}

Since current harmonics due to the converter switching and imperfect PWM are regarded as disturbances, and overmodulation non-linearity has been treated during the controller design stage, the transfer function described by Eq. (1) is used for simulations. This is a linear system with parameter uncertainties.

Figure 6 shows the step responses of the closed-loop system subject to parameter variations over the stipulated range, and confirms that the transient dynamic characteristics have been satisfied. The tracking performance can be assessed by considering the frequency response from the current reference demand to the current output, Figure 7 , together with three examples of time-domain responses at input frequencies of $60 \mathrm{~Hz}, 400 \mathrm{~Hz}$ and $1 \mathrm{kHz}$, to represent, respectively, drive applications in the industrial, aerospace and specialist market sectors, Figure 8(a), (b) and (c). It is observed that at the highest input frequency of $1 \mathrm{kHz}$, the phase shift is less than $30^{\circ}$, which is equivalent to a power factor of above $88 \%$ and is within the design specification. The control effort is also shown to lie within the design constraint of $-39 \mathrm{~dB}$ (or 0.011 ), Figure 9.

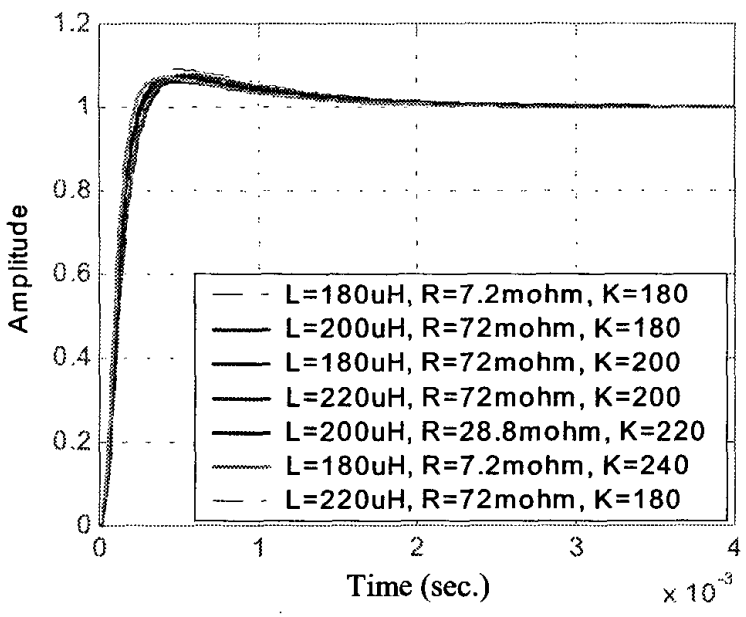

Figure 6. Step responses of closed-loop control system subject to parameter uncertainties

\section{B. Comparison with PI Control}

A PI controller may also be used for the current control, viz.

$$
G(s)=k_{p}+\frac{k_{i}}{s}
$$


In order to give an over-damped response, the poles of the closed-loop system should be placed along the negative real axis in the s-domain, i.e., at $s=-k_{i} / k_{p}$, and $s=-\omega$. Thus, the proportional and integral gains are derived as:

$$
\left\{\begin{array}{l}
k_{p}=\omega L / K \\
k_{i}=\omega R / K
\end{array}\right.
$$
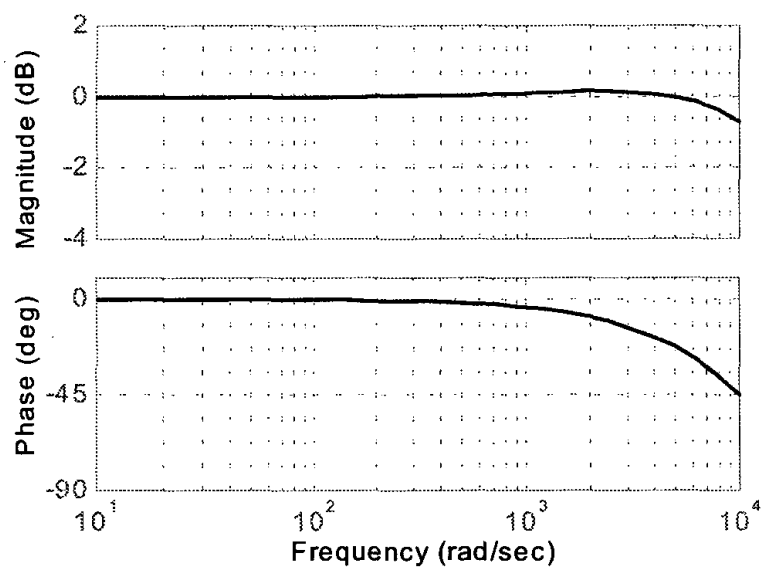

Figure 7. Frequency response of current tracking control system.
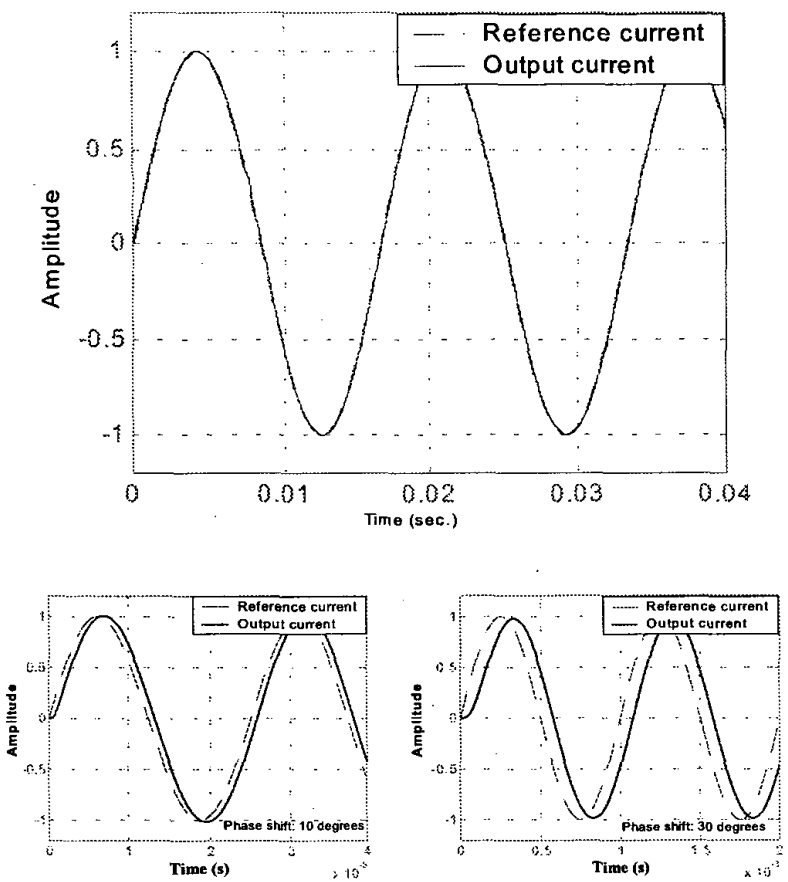

Figure 8. Time-domain current tracking performance. Input frequency: (a) $60 \mathrm{~Hz}$, (b) $400 \mathrm{~Hz}$, and (c) $1 \mathrm{kHz}$..

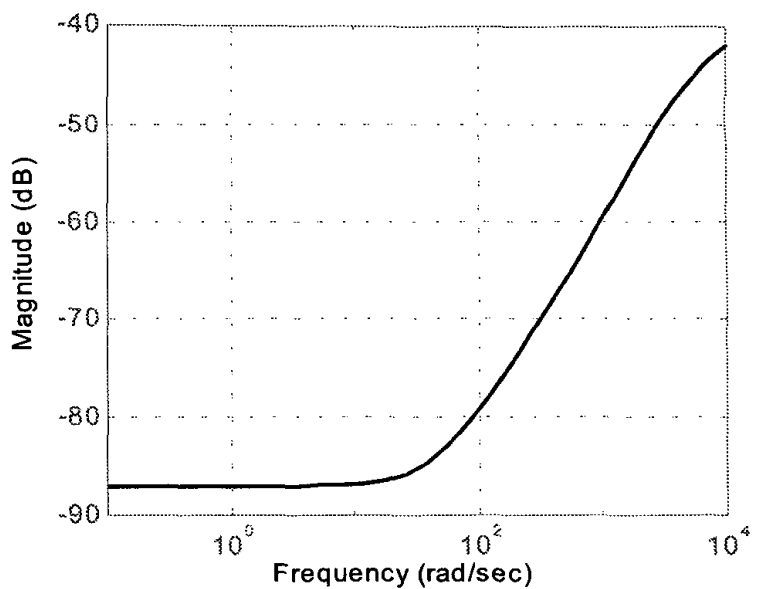

Figure 9. Control effort constraint. The magnitude is below the design limit of $-39 \mathrm{~dB}$ (or 0.011).

If the desired closed-loop pole is set at $\omega=10000 \mathrm{rad} / \mathrm{s}$ so as to provide a sufficiently short rise time, and the system parameters are selected at the nominal values given in Table 1, i.e., $L=180 \mu H, R=7.2 m \Omega$ and $K=180$, the PI gains are:

$$
\left\{\begin{array}{l}
k_{p}=1 \times 10^{-2} \\
k_{i}=0.4
\end{array}\right.
$$

Figure 10 shows that the resulting closed-loop system possesses a very good transient response when uncertainty is ignored. However, when system parameter variations are included, such as $L=220 \mu H, R=72 \mathrm{~m} \Omega$ and $K=180$, it can be readily shown that the system performance is significantly degraded, which was not the case with the QFT controller. As a result, there will be a steady-state error in current tracking, as shown in Figure 11.

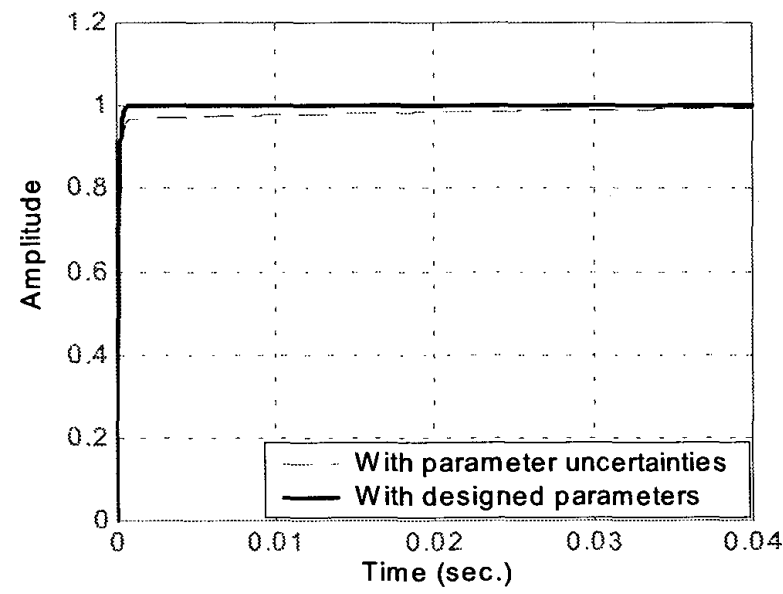

Figure 10. System step responses with PI control. 
An experimental facility to assess active filter performance has just been commissioned. Fuji IPM modules (7MBP) rated at $300 \mathrm{~A}, 600 \mathrm{~V}$ have been employed for the power converter, the measured three-phase voltages and currents being sampled by a DSP-based (TMS320C30) hardware development platform. The d.c. link voltage and current are also being monitored for the derivation of the desired reference current magnitude. The PWM switching is limited to $20 \mathrm{kHz}$.

Experiments are currently in progress, and will be reported in due course.

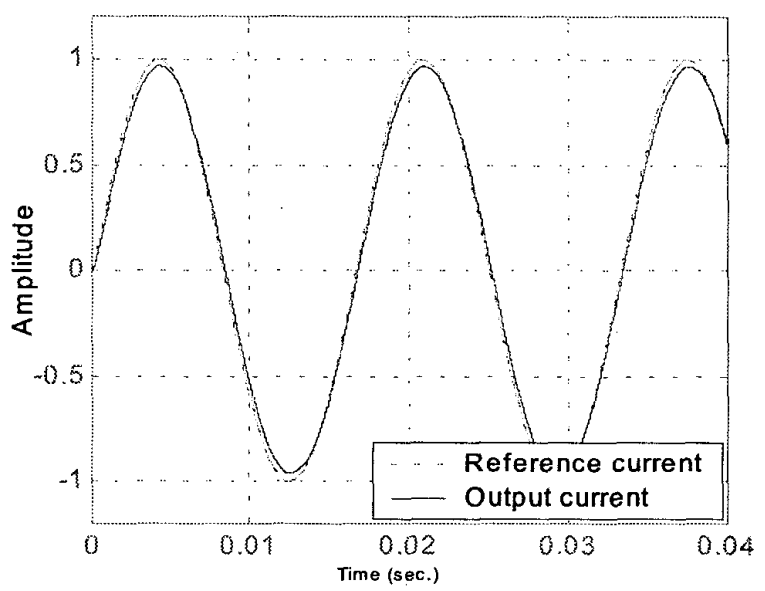

Figure 11. Current tracking performance with PI control. Input frequency: $60 \mathrm{~Hz}$.

\section{Conclusions}

The quality of the power utility, in terms of current harmonic content and power factor when supplying power electronic converter loads, can be improved by the application of active power filters. One of the primary issues of the system is the current tracking control, since ideally there should be no phase and amplitude errors between the reference and output currents over a wide frequency range. The paper has presented a new design methodology for robust current tracking control, which is based on quantitative feedback theory (QFT). The design requirements, in terms of tracking performance and disturbance rejection performance, have been specified, and design problems, which stem from parametric uncertainty due to line filter parameter variations, converter control over-modulation non-linearity, imperfections in the modulation algorithm, system output disturbances due to current harmonics and system input disturbances due to d.c. link voltage fluctuations, etc. have also been addressed in the design methodology. The performance attributes of the resulting controller have been verified by computer simulations, and the design has been shown to be superior to a 'classically' designed PI compensation scheme with regard to robustness and current tracking.

\section{REFERENCES}

[1] Sedighy M, Dewan S. B. and Dawson F. P. "A robust digital current control method for active power filters", IEEE Trans. on Industry Applications, Vol.36, No.4, 2000, pp. 1158-1164.

[2] Horowitz I. "Synthesis of feedback systems", Academic Press, New York, 1963.

[3] Horowitz I. "Survey of quantitative feedback theory (QFT)”, Int. J. Control, 53(2), 1991, pp. 255-291.

[4] Borhgesani C, Chait Y and Yaniv O. "Quantitative feedback theory toolbox", The MATH WORKS Inc. 1994.

[5] D’Azzo J. J. and Houpis C. H. "Linear control system analysis and design", McGraw-Hill Inc. 1995. 